\title{
Application of a Novel Small-Scale Sample Cleanup Procedure Prior to MALDI-TOF-MS for Rapid Pigment Fingerprinting of Red Wines
}

\author{
Violeta Ivanova Petropulos • Ágnes Dörnyei • Marina Stefova • \\ Trajče Stafilov • Borimir Vojnoski • László Márk • \\ Isidro Hermosín-Gutiérrez • Ferenc Kilár
}

Received: 17 May 2013 / Accepted: 17 July 2013 /Published online: 9 August 2013

(C) Springer Science+Business Media New York 2013

\begin{abstract}
This study evaluates the anthocyanin and derived pigment composition of Vitis vinifera red wines of Vranec, Merlot, and Cabernet Sauvignon produced in 2006, 2007, and 2008 vintages from the Tikveš wine region in the Republic of Macedonia. Their profile was established using matrix-assisted laser desorption/ionization time-of-flight mass spectrometry (MALDI-TOF-MS) technique. A total of 22 anthocyanins and derived pigments have been identified in the samples
\end{abstract}

V. Ivanova Petropulos $(\bowtie)$

Faculty of Agriculture, University Goce Delčev, Krste Misirkov bb, 2000 Štip, Republic of Macedonia

e-mail: violeta.ivanova@ugd.edu.mk

\section{Á. Dörnyei · F. Kilár}

Department of Analytical and Environmental Chemistry, Faculty of Sciences, University of Pécs, Ifjúság útja 6, 7624 Pécs, Hungary

\section{Á. Dörnyei • F. Kilár}

Institute of Bioanalysis, Faculty of Medicine, University of Pécs, Szigeti út 12, 7624 Pécs, Hungary

\section{Stefova • T. Stafilov}

Institute of Chemistry, Faculty of Natural Sciences and Mathematics, Ss. Cyril and Methodius University, Arhimedova 5, 1000 Skopje,

Republic of Macedonia

\section{B. Vojnoski}

Department of Enology, Institute of Agriculture, Ss. Cyril and

Methodius University, Blvd Aleksandar Makedonski bb,

1000 Skopje, Republic of Macedonia

L. Márk

Department of Biochemistry and Medical Chemistry, Faculty of Medicine, University of Pécs, Szigeti út 12, H-7624 Pécs, Hungary

\section{Hermosín-Gutiérrez}

Instituto Regional de Investigación Científica Aplicada (IRICA), Escuela de Ingenieros Agrónomos, Universidad de Castilla-La Mancha, Ronda de Calatrava 7, 13071 Ciudad Real, Spain including 10 anthocyanins, 1 ethyl-bridged flavanol-anthocyanin adduct, and 11 pyranoanthocyanins. MALDI-TOF-MS analysis was performed after solid-phase extraction of the wines by using, for the first time, the Zip-Tip ${ }^{\circledR} \mathrm{C} 18$ stationary phase, introducing a novel small-scale sample cleanup procedure prior to the rapid MALDI-TOF-MS fingerprinting of wine samples. 2',4',6'-Trihydroxyacetophenone (dissolved in acetonitrile/water $1: 1, v / v$ ) was used as a matrix. The qualitative screening of anthocyanins and derived pigments with MALDITOF-MS confirmed the presence of glucoside, acetylglucoside, and $p$-coumaroylglucoside derivatives of anthocyanins in the wine samples. Furthermore, pyranoanthocyanins formed by reactions of anthocyanins with pyruvic acid and acetaldehyde, as well as flavanol-pyranoanthocyanins and ethyl-bridged flavan-3-ol-anthocyanin adduct pigments have been detected in the samples.

Keywords Wine - Anthocyanins · Pyranoanthocyanins . Ethyl-bridged pigments $\cdot 2^{\prime}, 4^{\prime}, 6^{\prime}$-Trihydroxyacetophenone . MALDI-TOF-MS

\section{Introduction}

The color of wine is a value of quality and the most influential factor for choosing wine in a market. It is mainly determined by the composition and concentration of anthocyanins. The main anthocyanins in wines from Vitis vinifera grape varieties are 3-Oglucosides, 3-O-acetylglucosides, 3-O-p-coumaroylglucosides, and, to a lesser extent, 3-O-caffeoylglucosides of delphinidin, cyanidin, petunidin, peonidin and malvidin (Wulf and Nagel 1978; Ivanova et al. 2011a). During vinification, the concentration of monomeric anthocyanins, especially acylated anthocyanins, decreases as a result of the interaction of anthocyanins with 
themselves (self-association) or with other phenolic compounds (copigmentation) such as flavonols, flavan-3-ols, or phenolic acids (Wrolstad et al. 2005; Guadalupe and Ayestarán 2008; He et al. 2012). These reactions occur during the maceration processes and wine aging. They are followed by an irreversible formation of more complex and stable anthocyanin-derived pigments, such as pyranoanthocyanins, ethyl-bridged flavanol-anthocyanin adducts, and other pigments influencing wine expression such as in color, mouth feel, and sensory properties of red wine (Wrolstad et al. 2005; Monagas et al. 2005; Jensen et al. 2008).

Many studies have been performed on the structure and formation mechanisms of anthocyanin derivatives, as well as on the conditions that enable their formation (Berg and Akiyoshi 1975; Bakker and Timberlake 1986; Bakker and Timberlake 1997; Fulcrand et al. 1998; Alcalde-Eon et al. 2006). Different techniques have been used for pigment analysis and determination of their molecular masses. The most commonly used technique is high-performance liquid chromatography (HPLC), for the separation of the pigments, coupled to electrospray ionization mass spectrometry, as one of the most powerful techniques for structure characterization of wine components that is especially valuable for the identification of new derived wine pigments (Mateus et al. 2003; Kelebek et al. 2007; Jemal et al. 1998; Ivanova et al. 2011a). Furthermore, nuclear magnetic resonance or atmospheric pressure chemical ionization is also used for the characterization of phenolic compounds (Mateus et al. 2002, 2004; Ferrari et al. 2011).

In addition, matrix-assisted laser desorption/ionization time-of-flight mass spectrometry (MALDI-TOF-MS) is a powerful new technique that has a great potential in food analysis, as well as on wine and grape analysis (Sugui et al. 1998, 1999; Wang et al. 1999; Wang and Sporns 1999; Reed et al. 2005; Carpentieri et al. 2007; Ivanova et al. 2011b). This technique allows successful determination of the molecular weights in complex samples directly from ion abundances in the mass spectrum without previous isolation or cleanup of the sample (Hanton 2001; Ivanova et al. 2011b). The MALDI technique, for the first time, was demonstrated by Karas et al. 1987, originally being developed for the analysis of large biomolecules, such as proteins. MALDI-MS has also been applied to carbohydrates (Mock et al. 1991) and fructooligosaccharides in plants and food samples (Metzger et al. 1994; Stahl et al. 1997; Wang et al. 1999). In addition, MALDI-TOF-MS has been used for the identification of wine and grape anthocyanins and confirmation of the dominant anthocyanin compounds such as malvidin and its derivatives, using different matrices (2,5-dihydroxybenzoic acid (2,5-DHB), $\alpha$-cyano-4-hydroxycinnamic acid (CHCA), and sinapic acid (SA)), as well as $\mathrm{C}_{70}$ fullerene applied for the first time for this purpose, without sample preparation (Ivanova et al. 2011b). The advantages of MALDI-TOFMS over other methodologies include the ease of use, speed of analysis, high sensitivity, wide applicability combined with a good tolerance toward contaminants, as well as the ability to analyze complex mixtures such as wine (Stefova and Ivanova 2011).

The aim of this study was to test the applicability of the MALDI-TOF-MS methodology for fingerprinting the pigment profile of red wines after a fast and simple sample preparation procedure. The suitability of the method was demonstrated by performing a detailed anthocyanin and pigment profile determination of Vranec wine, the most widespread and typical variety for Macedonia and the Balkans, and Cabernet Sauvignon, and Merlot wines, as worldwidely known and popular varieties, all produced in Tikveš wine region in the Republic of Macedonia. The MALDI-TOF-MS technique was employed for fast fingerprinting of anthocyanins and derived pigments in wines after solid-phase extraction with Zip-Tip pipette tips, used for the first time for fast and efficient wine sample cleanup.

\section{Materials and Methods}

\section{Chemicals and Reagents}

$2^{\prime}, 4^{\prime}, 6^{\prime}$-Trihydroxyacetophenone was from Sigma-Aldrich (St. Louis, MO, USA). The standard of malvidin-3-glucoside was purchased from LGC Promochem GmbH, Szentendre (Hungary). Acetonitrile (HPLC-grade) was purchased from Scharlau Chemie S.A. All the other used reagents were of analytical purity grade. Zip-Tip ${ }^{\circledR}$ pipette tips filled with $\mathrm{C} 18$ stationary phase were from Millipore.

\section{Wine Samples}

In total, nine red wine samples from three $V$. vinifera varieties (Vranec, Merlot, and Cabernet Sauvignon) from three different vintages $(2006,2007$, and 2008) were subject of the investigation. All wine samples were kindly provided by Tikveš Winery, Kavadarci, Republic of Macedonia.

\section{Sample Cleanup by Solid-Phase Extraction Using Zip-Tip ${ }^{\circledR}$ C18 Pipette Tips}

For the extraction of wine pigments, a solid-phase extraction procedure was applied using reversed-phase Zip-Tip ${ }^{\circledR}$ pipette tips. The Zip-Tip ${ }^{\circledR} \mathrm{C} 18$ tips were washed with a $1-\mu \mathrm{L}$ mixture of acetonitrile/0.1 \% $(v / v)$ and trifluoroacetic acid (TFA) in water $(1 / 1, v / v)$ and conditioned with $1 \mu \mathrm{L}$ of $0.1 \%$ TFA in water. Then, the wine sample $(1 \mu \mathrm{L})$ was aspirated and dispensed through the tip. One microliter of $0.1 \%(v / v)$ TFA in water was used to remove the sugars and acids which were not retained by the $\mathrm{C} 18$ stationary phase under these conditions. Elution of pigments from the stationary phase was performed 
with a $1-\mu \mathrm{L}$ solution mixture of acetonitrile $/ 0.1 \%(v / v)$ TFA in water $(1 / 1, v / v)$.

\section{MALDI-TOF-MS and MS/MS Analyses}

The matrix solution was prepared by dissolving $10 \mathrm{mg}$ of 2', $4^{\prime}, 6^{\prime}-$ trihydroxyacetophenone (THAP) in $1 \mathrm{~mL}$ of acetonitrile/ water $(1 / 1, v / v)$. After the solid-phase extraction with Zip-Tip ${ }^{\circledR}$ $\mathrm{C} 18$ tips, $1 \mu \mathrm{L}$ of the eluate was mixed with $1 \mu \mathrm{L}$ of the matrix solution on the target plate (MTP 384 massive target plate; Bruker Daltonics, Bremen, Germany). After sample crystallization, the target plate was introduced into an Autoflex II MALDI-TOF/TOF MS instrument (Bruker, Daltonics, Bremen, Germany). The samples with the matrix were ionized by nitrogen laser pulse $(\lambda=337 \mathrm{~nm}, 50 \mathrm{~Hz})$. The laser power was adjusted between 20 and $30 \%$ of its maximum intensity. Pulsed ion extraction was applied with 80 ns of delay; ion source voltages 1 and 2 were 19 and $17.05 \mathrm{kV}$, and the ion source lens voltage was set to $8.2 \mathrm{kV}$.

Mass spectrometry data were acquired in the positive ionization mode and reflectron operation mode; the reflectron voltage was set to $+20 \mathrm{kV}$. The mass spectra were recorded in the $m / z$ ranges of $350-1,400$ or $750-1,800$ and were at the sum of 300 consecutive laser shots on a sample spot. The precursor isolation window for the MS/MS experiments was set to $\pm 1 \%$ of the mass of the precursor ion. Data processing was performed using FlexAnalysis 2.4 software package (Bruker Daltonics, Bremen, Germany).

\section{Results and Discussion}

The primary aim of this study was to develop and utilize a novel small-scale sample cleanup procedure that will be applied prior to the MALDI-TOF-MS for rapid fingerprinting of wine samples. Previously, we had used the MALDI-TOFMS technique for fingerprinting purposes in case of wine and grape samples, and we had tested the applicability of several matrices including 2,5-dihydroxybenzoic acid (2,5-DHB), $\alpha$ cyano-4-hydroxycinnamic acid, sinapic acid (SA), as well as $\mathrm{C}_{70}$ fullerene. 2,5-DHB was superior for the identification only of the main anthocyanins in wine and grape (Ivanova et al. 2011b), opposite of previously published results (Carpentieri et al. 2007) where different kinds of chemical compounds were detected in wine samples using this matrix. Furthermore, $\mathrm{C}_{70}$ fullerene was applied for the first time for wine analysis with MALDI-TOF-MS, without any prior sample cleanup procedure (Ivanova et al. 2011b), giving good quality spectra and matrix peaks with very low intensities in range of $\mathrm{m} / \mathrm{z} 100$ 700. Since a low number of pigments in wine were detected using the 2,5-DHB and $\mathrm{C}_{70}$ fullerene matrices in the previous study and in order to be able to detect more anthocyanins, as well as derived pigments in wine, a sample cleanup, including the extraction of these compounds from the complex matrix, is necessary to be performed. Therefore, the solid-phase extraction with Zip-Tip ${ }^{\circledR} \mathrm{C} 18$ pipette tips has been used for the extraction of anthocyanins and derived pigments from the wine samples in this study. Zip-Tip C18 is a $10-\mu \mathrm{L}$ pipette tip with a micro-volume $(0.5 \mu \mathrm{L})$ bed of reversed-phase medium fixed at its end, i.e., a miniature reversed-phase column. This type of tip was chosen since it was considered that it would be appropriate for purifying and concentrating the wine samples in order to provide better data quality. Furthermore, the whole extraction procedure (described in

Table 1 Phenolic compounds found in Vranec, Cabernet Sauvignon, and Merlot wines identified by MALDI-TOF-MS analysis

\begin{tabular}{lll}
\hline Phenolics & MS $(\mathrm{m} / \mathrm{z})$ & $\begin{array}{l}\text { MS/MS }(\mathrm{m} / \mathrm{z}) \\
\text { Fragment ions }\end{array}$ \\
& $\mathrm{M}^{+}$ & \\
\hline Anthocyanins & & \\
Non-acylated glucosides & & \\
Dp-3-glc & 465 & 303 \\
Pt-3-glc & 479 & 317 \\
Pn-3-glc & 463 & 301 \\
Mv-3-glc & 493 & 331 \\
Acetylglucosides & & \\
Mv-3-acetylglc & 535 & 331 \\
p-Coumaroylglucosides & & \\
Dp-3-p-coumglc & 611 & 303 \\
Cy-3-p-coumglc & 595 & 287 \\
Pt-3-p-coumglc & 625 & 317 \\
Pn-3-p-coumglc & 609 & 301 \\
Mv-3-p-coumglc & 639 & 331 \\
Ethyl-bridged flavan-3-ol-anthocyanin adducts & \\
(Epi)cat-ethyl-Mv-3-p-coumglc & 955 & 665,357 \\
Pyranoanthocyanins & & \\
Vitisin A & 561 & 399 \\
p-Cm-vitisin A & 707 & 399 \\
Vitisin B & 517 & 355 \\
Ac-vitisin B & 559 & 355 \\
$p$-Cm-vitisin B & 663 & 355 \\
A-type vitisin of Pt-3-p-coumglc & 677 & 369 \\
10-DHP-pymv-3-glc (pinotin A) & 625 & 463 \\
10-MHP-pymv-3-cmglc & 755 & 447 \\
10-(Epi)Cat-pymv-3-glc & 805 & 643,491 \\
10-(Procyanidin dimer)-pymv-3-glc & 1,093 \\
10-(Procyanidin dimer)-pymv-3-acglc & 1,135 \\
\hline
\end{tabular}

The details on the MALDI-TOF-MS are described in the "MALDI-TOFMS and MS/MS Analyses" section

$D p$ delphinidin, $C y$ cyanidin, $P t$ petunidin, $P n$ peonidin, $M v$ malvidin, Cat catechin, Epicat epicatechin, glc 3-glucoside, acglc 3-(6"-acetyl)-glucoside, cmglc 3-(6"-coumaroyl)-glucoside, 10-MHP 10-(4"'-monohydroxyphenyl), 10-DHP 10-(3"', 4 "'-dihydroxyphenyl), pymv pyranomalvidin, vitisin A 10carboxy-pyrmv-3-glc, vitisin B 10-H-pymv-3-glc, A-type vitisin 10 carboxy-pyranoanthocyanins 
Fig. 1 Positive-ion MALDITOF mass spectra of Vranec wine, produced in 2008. $\mathrm{m} / \mathrm{z}$ values of identified compounds are presented in Table 1

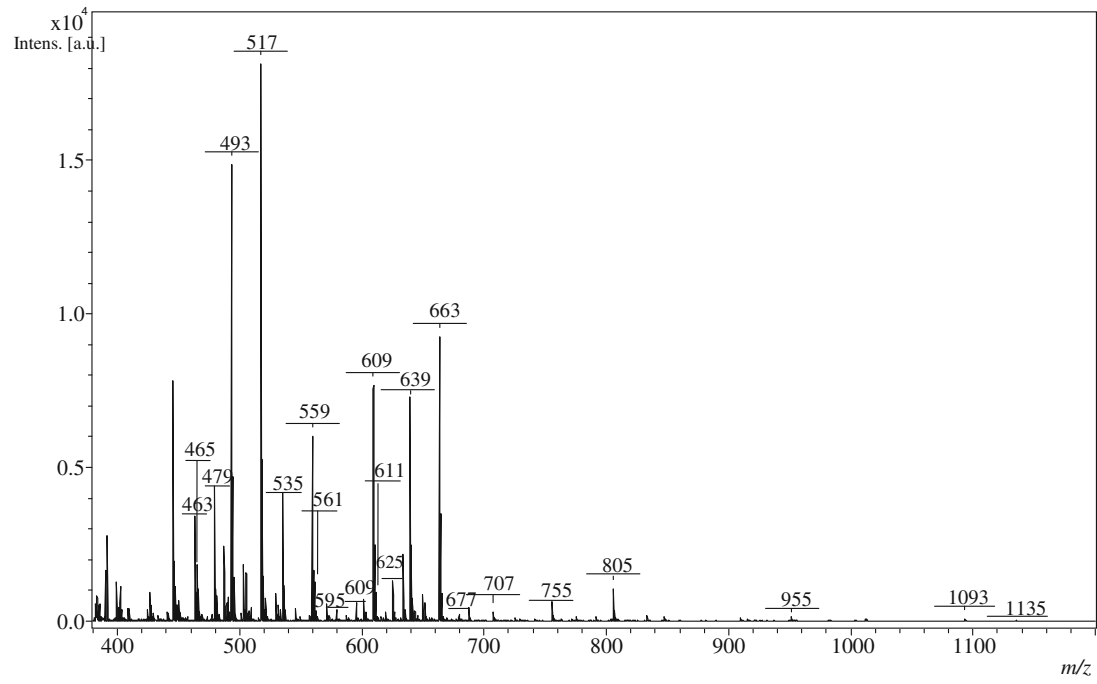

the "Sample Cleanup by Solid-Phase Extraction Using ZipTip ${ }^{\circledR}$ C18 Pipette Tips" section) required only few minutes and small volumes $(1 \mu \mathrm{L})$ of solvents (acetonitrile and $0.1 \%$ $(v / v)$ TFA in water).

As for the choice of matrix, THAP has been used as convenient matrix for the analysis of oligosaccharides and polyphenols in food samples (Wang et al. 1999), giving small homogeneous crystals and better resolution of the glucosides. THAP was also successfully used as a matrix for peptide and oligonucleotide analysis (Kussmann et al. 1997). Applying this matrix for wine analysis, homogeneous sample preparation and high ionization efficiency were achieved, confirming its suitability for wine phenolic compound identification. Thus, the proposed MALDI-TOF-MS analysis combined with solid-phase extraction using Zip-Tip ${ }^{\circledR} \mathrm{C} 18$ pipettes and THAP matrix provided a rapid method for qualitative screening of wine pigments, taking only few minutes in total and greatly

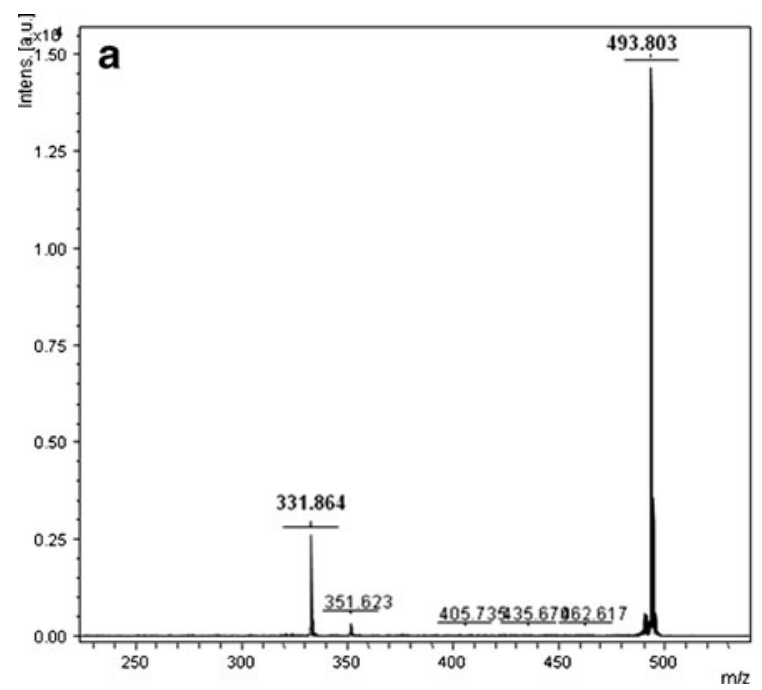

reducing the analysis time compared to traditional HPLCDAD-MS methods.

The solid-phase extraction method with Zip-Tip ${ }^{\circledR}$ C18 pipettes was applied for wine cleanup, and in total, 22 compounds have been tentatively identified by MALDI-TOF-MS technique. Table 1 and Fig. 1 present the identified compounds, belonging to five groups: anthocyanins, vitisin-type pyranoanthocyanins, hydroxyphenyl-pyranoanthocyanins, flavanyl-pyranoanthocyanins, and acetaldehyde-mediated flavanol-anthocyanin adducts. The identification was performed on the basis of the monoisotopic molecular mass of the flavylium cations $\left(\mathrm{M}^{+}\right.$signals) or the protonated quasimolecular ions $\left([\mathrm{M}+\mathrm{H}]^{+}\right.$signals) under positive ionization mode and confirmed by MS/MS fragmentation. The identification was additionally confirmed using the standard of malvidin-3glucoside analyzed under the same experimental conditions and/or with comparison of the results in already published

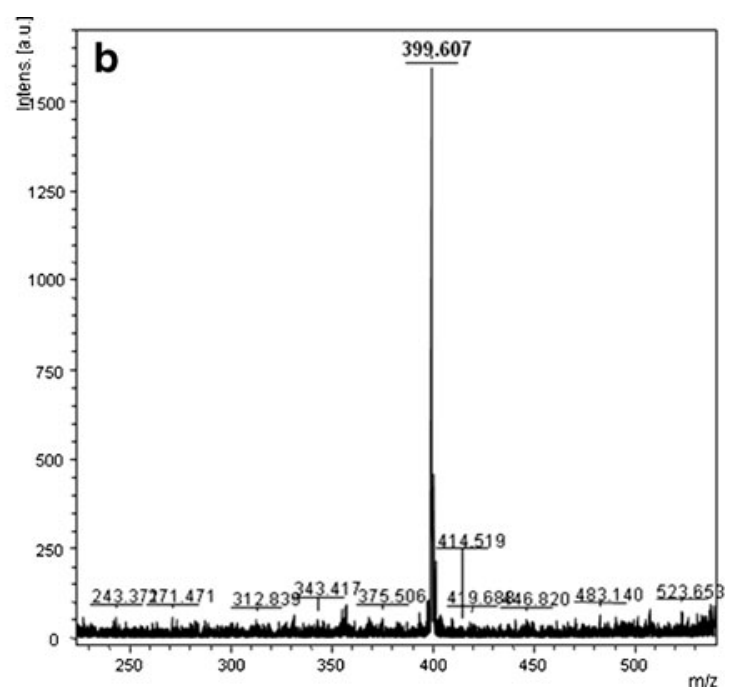

Fig. 2 MALDI-MS/MS fragmentation of the ions at $m / z 493$ (a) and $m / z 707$ (b) under positive mode 

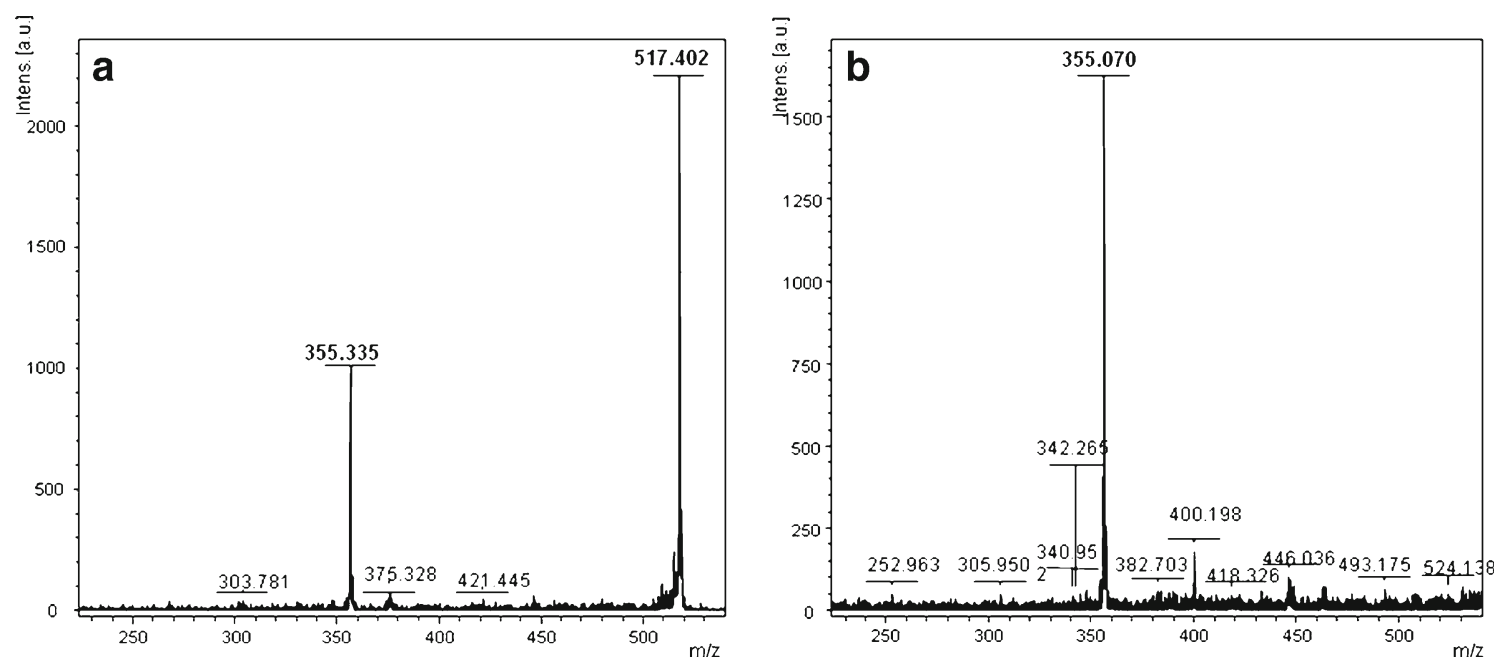

Fig. 3 MALDI-MS/MS fragmentation of the ions at $m / z 517$ (a) and $m / z 559$ (b) under positive mode

data. Thus, the cation of malvidin-3-glucoside $\left(\mathrm{M}^{+}\right)(\mathrm{m} / \mathrm{z} 493)$ was detected in all samples. The molecular ion peaks that appeared in the mass spectrum, at $\mathrm{m} / \mathrm{z} 535$ and 639, corresponded to the malvidin-3-acetylglucoside and malvidin3-p-coumaroylglucoside, respectively. The fragmentation of these three ions $(\mathrm{m} / \mathrm{z} 493,535$, and 639) produced fragment ion at $\mathrm{m} / \mathrm{z} 331$ as a result of the elimination of glucose, acetylglucoside, and $p$-coumaroylglucoside moieties, respectively (Ivanova et al. 2011a). Molecular peaks at $\mathrm{m} / \mathrm{z}$ 465, 479, and 463 in the MALDI-TOF-MS spectrum of the wine extract were identified as 3-glucosides of delphinidin, petunidin, and peonidin, respectively. 10-Carboxy-pyranoanthocyanins or A-type vitisins (vitisin A and $p$-coumaroyl-vitisin A) were detected on the basis of their $\mathrm{M}^{+}$signals at $m / z 561$ and 707, respectively, giving fragment at $\mathrm{m} / \mathrm{z} 399$, corresponding to the loss of glucose $(162 \mathrm{u})$ and $p$-coumaroylglucoside $(308 \mathrm{u})$ moieties, respectively. The molecular ion peak at $m / z 677$ and a fragment ion at $\mathrm{m} / \mathrm{z} 369$ were identified as A-type vitisin of petunidin-3-p-coumaroylglucoside (Blanco-Vega et al. 2011). The MALDI-MS/MS fragmentation of malvidin-3-glucoside
( $m / \mathbf{z} 493)$ and $p$-coumaroyl-vitisin A $(m / z$ 707) is presented in Fig. 2.

Another group of wine-derived pigments has been identified belonging to the group of 10-H-pyranoanthocyanins (B-type vitisins, or simply pyranoanthocyanins). Molecular ion peaks at $\mathrm{m} / \mathrm{z} 517,559$, and 663 were identified as pyranomalvidin-3-glucoside (vitisin B), pyranomalvidin-3acetylglucoside (acetyl-vitisin B), and pyranomalvidin-3-pcoumaroylglucoside ( $p$-coumaroyl-vitisin B), all producing a fragment ion at $\mathrm{m} / \mathrm{z} 355$ as a result of the elimination of glucoside $(162 \mathrm{u})$, acetylglucoside $(204 \mathrm{u})$ and $p$-coumaroylglucoside (308 u) groups, respectively (Ivanova et al. 2011a; Blanco-Vega et al. 2011). The MALDI-MS/MS fragmentation of vitisin B $(\mathrm{m} / \mathrm{z}$ $517)$ and acetyl-vitisin B ( $/ \mathrm{z} 559)$ is presented in Fig. 3.

In addition, three 10-flavanyl-pyranoanthocyanin pigments have been detected in the wines, showing signals at $\mathrm{m} / \mathrm{z} 805$ (fragment ions $\mathrm{m} / \mathrm{z}$ 643, 491), $\mathrm{m} / \mathrm{z} 1,093$ (fragment ions $m / z$ 931, 803), and $m / z 1,135$ (fragment ions $m / z$ 931, 845). These pigments referred to 10-(epi)catechin-pyranomalvidin-3glucoside, 10-(procyanidin dimer)-pyranomalvidin-3-glucoside,
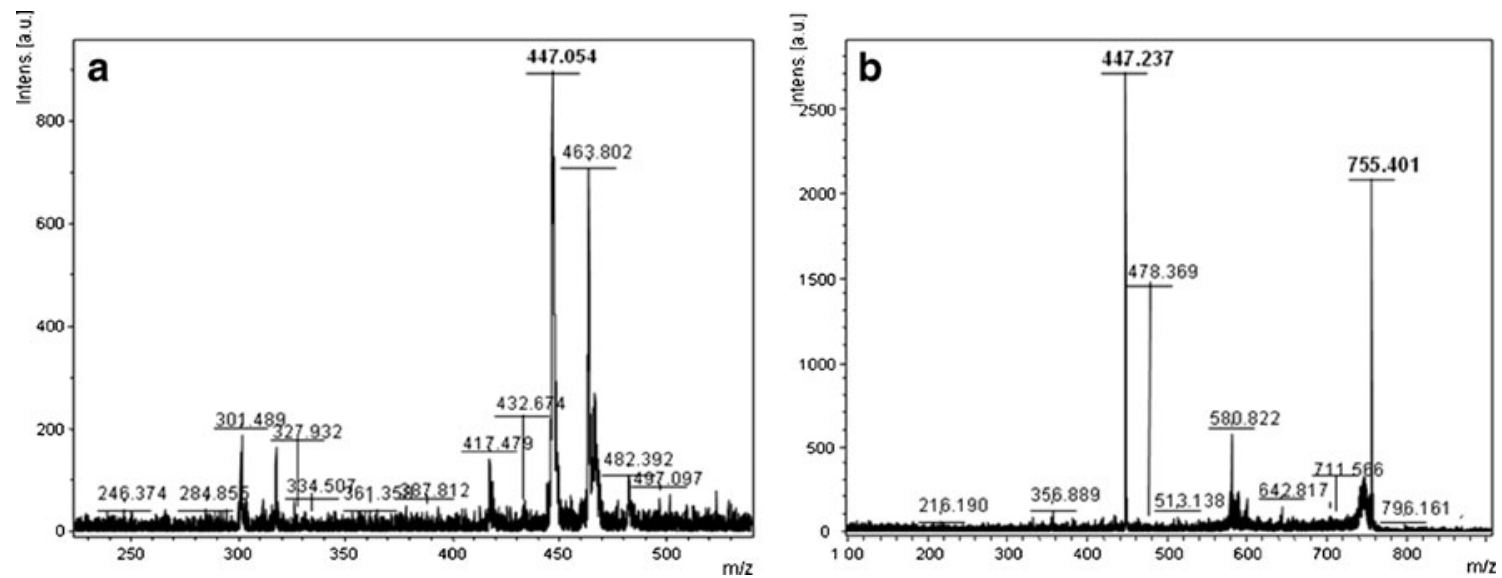

Fig. 4 MALDI-MS/MS fragmentation of the ions at $m / z 625$ (a) and $m / z 755$ (b) under positive mode 
Fig. 5 Positive-ion MALDITOF-MS mass "fingerprint" spectra of Vranec wines (2006, 2007, and 2008 years of production) obtained after SPE with Zip-Tip C18 pipette tips. $\mathrm{m} / \mathrm{z}$ values of the most abundant identified compounds are presented in Table 1

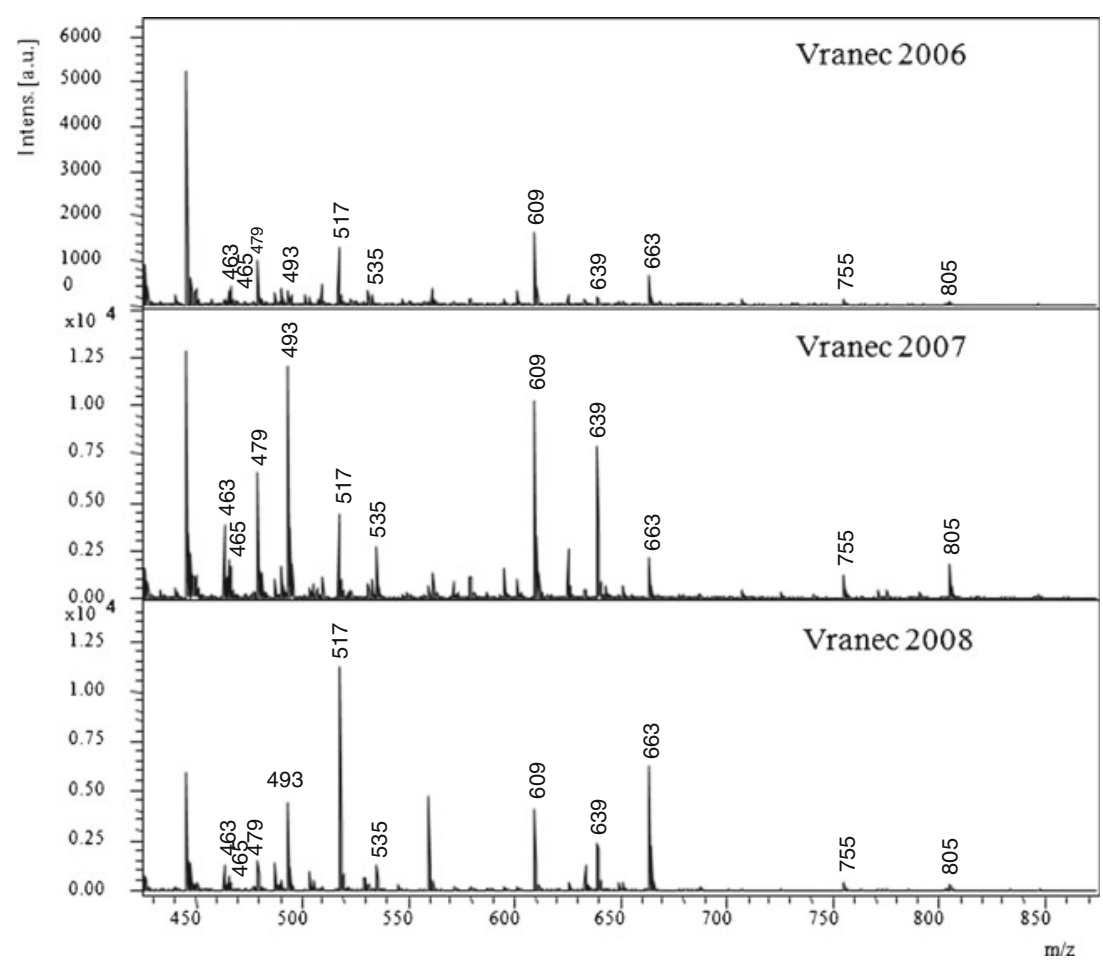

and 10-(procyanidin dimer)-pyranomalvidin-3-p-acetylglucoside, respectively. Fragmentation of the first molecular ion $(\mathrm{m} / \mathrm{z} 805)$ yielded aglycone cation at $m / z 643$ as a result of loss of glucoside, and the molecular ions at $m / z 1,093$ and 1,135 yielded cations at $\mathrm{m} / \mathrm{z} 931$ as a result of the elimination of acetylglucoside groups.
The fragment ion at $m / z 491$ corresponded to a retro-Diels-Alder fission of the (+)-catechin moiety (152 U). The fragments at $\mathrm{m} / \mathrm{z}$ 803 and 845, for both components, respectively, were formed by the cleavage of interflavonoid bond of procyanidin dimers producing loss of flavan-3-ol monomer ion followed by
Fig. 6 Positive-ion MALDITOF-MS mass "fingerprint" spectra of Vranec, Merlot, and Cabernet Sauvignon wines, vintage 2006, obtained after SPE with Zip-Tip C18 pipette tips. $\mathrm{m} / \mathrm{z}$ values of the most abundant identified compounds are presented in Table 1

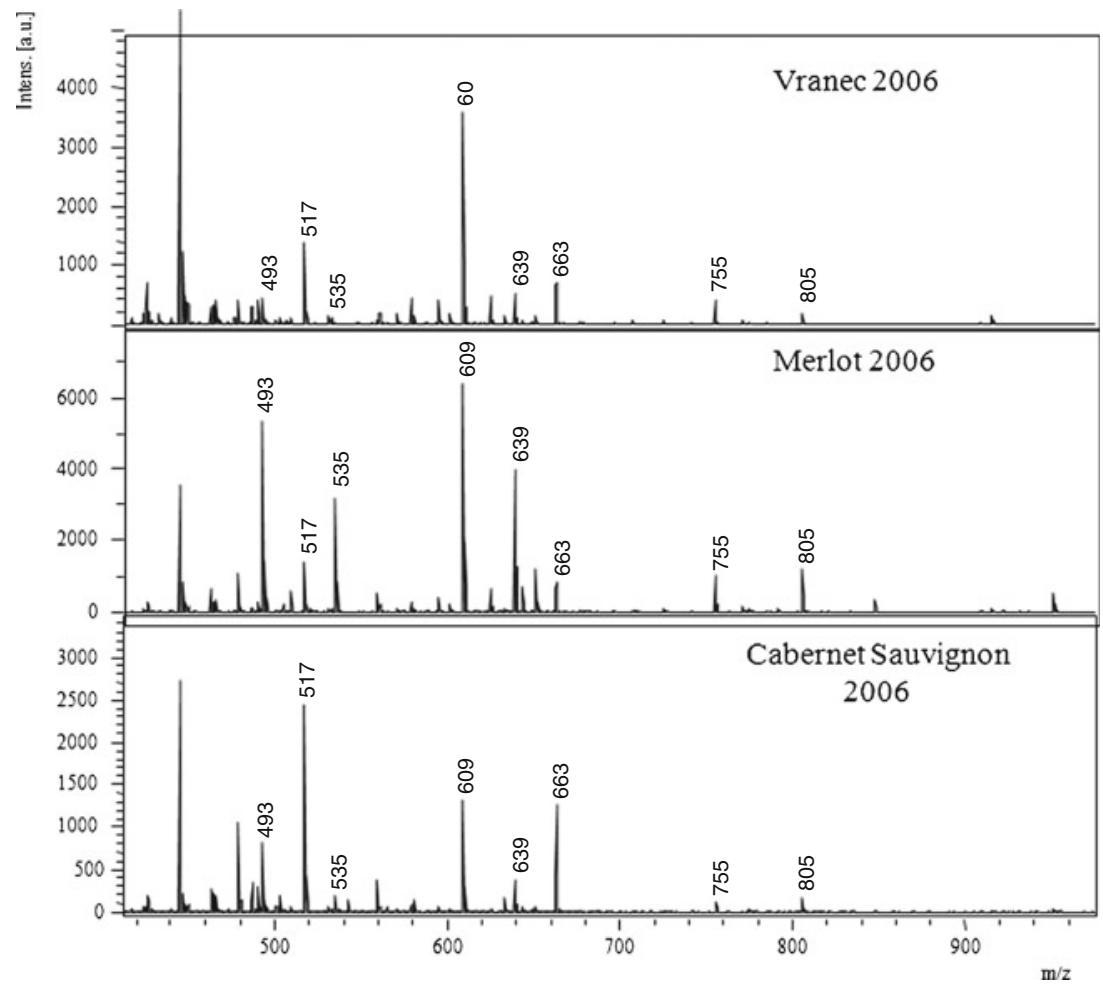


formation of flavanyl-carboxy-pyranomalvidin-3-glucoside and flavanyl-carboxy-pyranomalvidin-3-acetylglucoside ions, respectively. One acetaldehyde-mediated flavanol-anthocyanin condensed product has been also detected in the wines. This compound was identified as (epi)catechin-ethyl-malvidin-3- $p$ coumaroylglucoside, presenting a molecular ion at $\mathrm{m} / \mathrm{z} 955$ in the MALDI-TOF-MS spectrum. Fragmentation of the molecular ion produced fragment ions at $m / z 665$ and 357 . The first fragment $(m / z 665)$ corresponds to the elimination of an (epi)catechin molecule $(\mathrm{m} / \mathrm{z} 290)$, and the second fragment is a result of loss of $p$-coumaroylglucoside group.

Two compounds belonging to the group of hydroxyphenylpyranoanthocyanins were detected in wines: the compound with molecular ion at $m / z 625$ and fragment ion at $m / z 463$ was identified as $10-\left(3^{\prime \prime \prime}, 4^{\prime \prime \prime}\right.$-dihydroxyphenyl)-pyranomalvidin-3glucoside, also known as pinotin A (Rentzsch et al. 2010) and the compound with molecular ion at $m / z 755$ (fragment ions at $\mathrm{m} / \mathrm{z} 447)$ was identified as 10-(4"'--monohydroxyphenyl)pyranomalvidin-3-p-coumaroylglucoside. Figure 4 shows the MALDI-MS/MS fragmentation of compounds with $\mathrm{m} / \mathrm{z} 625$ and 755 .

Figure 5 presents the positive-ion MALDI-TOF-MS mass "fingerprint" spectra of Vranec wines from different vintages. The relative abundance of the mass signals of the main anthocyanin, malvidin-3-glucoside, at $\mathrm{m} / \mathrm{z} 493$ is clearly different in all three samples, the highest abundance being observed in the wine produced in 2007. Similarly, the relative abundance of the mass signals at $\mathrm{m} / \mathrm{z} 535, \mathrm{~m} / \mathrm{z} 609$, and $\mathrm{m} / \mathrm{z} 639$, attributed to malvidin-3-acetylglucoside, peonidin-3-p-coumaroylglucoside, and malvidin-3-p-coumaroylglucoside, respectively, were highest in Vranec wine from 2007, which means that not only aging conditions but also oenological practices applied for winemaking influence the content of anthocyanins in wine, as well as the edaphoclimatic conditions where the vineyards are located, and the cultural practices applied to the vine plants. Figure 6 shows the MALDI mass spectrum of Vranec, Merlot, and Cabernet Sauvignon wines from same vintage (2006). It could be clearly noticed that anthocyanins and other pigments were present with highest abundance in Merlot wine.

As a conclusion, solid-phase extraction with Zip-Tip ${ }^{\circledR}$ pipette tips is a very fast method (requires at about $1 \mathrm{~min}$ of preparation) with very low solvent consumption (only few microliters) that simplifies sample and spectra, followed by rapid and simple MALDI-TOF-MS identification of a big number of wine pigments. This confirms the ability of MALDI-TOF-MS as a valuable technique for fast screening of wine samples.

\section{Conclusion}

Identification of anthocyanins and derived pigments was performed with the MALDI-TOF-MS technique. A new smallscale sample cleanup procedure using solid-phase extraction prior to the rapid MALDI-TOF-MS fingerprinting of wine samples was introduced, using $2^{\prime}, 4^{\prime}, 6^{\prime}$-trihydroxyacetophenone as a matrix. Twenty-two color compounds were detected in the wines, developing a rapid and simple MALDI method for wine analysis and confirming the ability of this technique for fast wine screening for the detection of glucoside, acetylglucoside, and $p$ coumaroylglucoside derivatives of anthocyanins as well as pyranoanthocyanins, flavanol-pyranoanthocyanins, and ethylbridged flavan-3-ol-anthocyanin adduct pigments. Using this analytical methodology, hydroxyphenyl-pyranoanthocyanins were, for the first time, identified in Macedonian wines.

Acknowledgments This work was financially supported by the Central European Exchange Program for University Studies (CEEPUS) Program realized through the CEEPUS network (CIII-HU-0010-06-Teaching and Learning Bioanalysis) covering the study stay of Violeta Ivanova Petropulos at the University of Pécs, Hungary. Á. D. acknowledges the support of the János Bolyai Research Scholarship (Hungarian Academy of Sciences). The authors express gratitude to Mr. Kire Trajkov from Tikveš Winery, Kavadarci, Republic of Macedonia, for providing the wine samples.

Conflict of Interest Violeta Ivanova Petropulos declares that she has no conflict of interest. Ágnes Dörnyei declares that she has no conflict of interest. Marina Stefova declares that she has no conflict of interest. Trajče Stafilov declares that he has no conflict of interest. Borimir Vojnoski declares that he has no conflict of interest. László Márk declares that he has no conflict of interest. Isidro Hermosín-Gutiérrez declares that he has no conflict of interest. Ferenc Kilár declares that he has no conflict of interest. This article does not contain any studies with human or animal subjects.

\section{References}

Alcalde-Eon C, Escribano-Bailón MT, Santos-Buelga C, Rivas-Gonzalo JC (2006) Anal Chim Acta 563:238

Bakker J, Timberlake CF (1986) Am J Enol Viticult 37:288

Bakker J, Timberlake CF (1997) J Agric Food Chem 45:35

Berg HW, Akiyoshi MA (1975) Am J Enol Vitucult 26(3):134

Blanco-Vega D, López-Bellido FJ, Alía-Robledo JM, Hermosín-Gutiérrez I (2011) J Agric Food Chem 59:9523

Carpentieri A, Marino G, Amoresano A (2007) Anal Bioanal Chem 389:969

Ferrari E, Foca G, Vignali M, Tassi L, Ulrici A (2011) Anal Chim Acta 701:139

Fulcrand H, Benabdeljalil C, Rigaud J, Cheynier V, Mountounet M (1998) Phytochem 47:1401

Guadalupe Z, Ayestarán B (2008) Eur Food Res Technol 228:29

Hanton SD (2001) Chem Rev 101:521

He F, Liang NN, Mu L, Pan QH, Wang J, Reeves MJ, Duan CQ (2012) Molecules 17:1483

Ivanova V, Dörnyei Á, Márk L, Vojnoski B, Stafilov T, Stefova M, Kilár F (2011a) Food Chem 124:316

Ivanova V, Dörnyei Á, Stefova M, Stafilov T, Vojnoski B, Kilár F, Márk L (2011b) Food Anal Method 4:108

Jemal M, Ouyang Z, Teitz DS (1998) Rapid Commun Mass Spectrom $12: 429$

Jensen JS, Demiray S, Egebo M, Meyer AS (2008) J Agric Food Chem 56:1105

Karas M, Bachmann D, Bahr U, Hillenkamp F (1987) Int J Mass Spectrom Ion Processes 78:53 
Kelebek H, Canbas A, Selli S (2007) Chromatographia 66:207

Kussmann M, Nordhoff E, Rahbek-Nielsen H, Haebel S, Rossel-Larsen M, Jakobsen L, Gobom J, Mirgorodskaya E, Kroll-Kristensen A, Palm L, Roepstorff P (1997) J Mass Spectrom 32:593

Mateus N, Carvalho E, Carvalho ARF, Melo A, Gonzlez-Params AM, Santos-Buelga C, Silva AMS, de Freitas V (2003) J Agric Food Chem 51:277

Mateus N, Oliveira J, Santos-Buelga C, Silva AM, de Freitas V (2004) Tetrahedron Lett 45:3455

Mateus N, Silva AM, Santos-Buelga C, Rivas-Gonzalo JC, de Freitas V (2002) J Agric Food Chem 50:2110

Metzger JO, Woisch R, Tuszynski W, Angermann R (1994) Fresen J Anal Chem 349:473

Mock KK, Daevy M, Cottrell JS (1991) Biochem Bioph Res Com 177:644

Monagas M, Bartolomé B, Gómez-Cordovés C (2005) Eur Food Res Technol 220:331
Reed JD, Krueger CG, Vestling MM (2005) Phytochemistry 66:2248

Rentzsch M, Schwarz M, Winterhalter P, Blanco-Vega D, HermosínGutiérrez I (2010) Food Chem 119:1426

Stahl B, Lions A, Karas M, Hillenkamp F, Steup M (1997) Anal Chem 246:1195-1204

Stefova M, Ivanova V (2011c) Analytical methodology for characterization of grape and wine phenolic bioactives, chapter 20. In: Fruits and cereal bioactives: sources, chemistry, and applications. CRC, Boca Raton, p409

Sugui JA, Bonham C, Lo SC, Wood KV, Nicholson RL (1998) Phytochemistry 8:1063

Sugui JA, Wood KV, Yang ZY, Bonham CC, Nicholson RL (1999) Am J Enol Viticult 50:199

Wang J, Sporns P, Low NH (1999) J Agric Food Chem 47:1549

Wang J, Sporns P (1999) J Agric Food Chem 47:2009

Wrolstad RE, Durst RW, Lee J (2005) Trends Food Sci Tech 16:423

Wulf LW, Nagel CW (1978) Am J Enol Viticult 29:42 